OPEN ACCESS

Edited by:

Qingxin Mu,

University of Washington,

United States

Reviewed by:

Hamed Mirzaei,

Kashan University of Medical

Sciences, Iran

Peifeng Li,

Qingdao University, China

*Correspondence:

Gang Chen

chengang@gxmu.edu.cn

Specialty section:

This article was submitted to

Cancer Molecular Targets and Therapeutics,

a section of the journal

Frontiers in Oncology

Received: 19 September 2020 Accepted: 18 December 2020 Published: 17 February 2021

Citation:

Xiong D, He R, Dang Y, Wu H, Feng Z and Chen $G$ (2021) The Latest

Overview of circRNA in the Progression, Diagnosis, Prognosis,

Treatment, and Drug Resistance of Hepatocellular Carcinoma.

Front. Oncol. 10:608257. doi: 10.3389/fonc.2020.608257

\section{The Latest Overview of circRNA in the Progression, Diagnosis, Prognosis, Treatment, and Drug Resistance of Hepatocellular Carcinoma}

\author{
Dandan Xiong ${ }^{1}$, Rongquan $\mathrm{He}^{2}$, Yiwu Dang ${ }^{1}$, Huayu $\mathrm{Wu}^{3}$, Zhenbo Feng ${ }^{1}$ \\ and Gang Chen ${ }^{1 *}$ \\ ${ }^{1}$ Department of Pathology, First Affiliated Hospital of Guangxi Medical University, Nanning, China, ${ }^{2}$ Department of Medical \\ Oncology, First Affiliated Hospital of Guangxi Medical University, Nanning, China, ${ }^{3}$ Department of Cell Biology \& Genetics, \\ School of Preclinical Medicine, Guangxi Medical University, Nanning, China
}

Hepatocellular carcinoma $(\mathrm{HCC})$ is one of the main causes of tumor-related deaths worldwide. Due to the lack of obvious early symptoms and the lack of sensitive screening indicators in the early stage of HCC, the vast majority of patients are diagnosed with advanced or metastatic HCC, resulting in dissatisfactory treatment result. Therefore, it is urgent to determine effective and sensitive diagnostic and prognostic indicators and to determine new therapeutic targets. Circular RNA (circRNA) is a type of non-coding RNA that has been neglected for a long time. In recent years, it has been proved to play an important role in the development of many human diseases. Increasing evidence shows that change in circRNA expression has an extensive effect on the biological behavior of HCC. In this study, we comprehensively tracked the latest progress of circRNA in the pathogenesis of $\mathrm{HCC}$, and reviewed its role as a biomarker for diagnosis and prognosis prediction in patients with HCC. In addition, we also summarized the potential of circRNA as therapeutic target in $\mathrm{HCC}$ and its relationship with $\mathrm{HCC}$ drug resistance, providing clues for the clinical development of circRNA-based therapeutic strategies.

Keywords: hepatocellular carcinoma, circular RNAs, biological function, biomarker, treatment, drug resistance

\section{INTRODUCTION}

Hepatocellular carcinoma (HCC) is the fourth leading cause of tumor-related deaths worldwide, causing approximately 78,200 deaths per year. In addition, it is estimated that there are 841,000 new cases of HCC worldwide each year, ranking seventh among all malignancies (1). Although treatment is improved in recent years, patients with HCC suffer unfavorable prognosis. The main reason is that the symptoms in early HCC are not obvious and there is a lack of practical

\footnotetext{
Abbreviations: AUC, area under the curve; circRNA, cicular RNA; ciRNA, circular intronic RNA; ecircRNA, exonic circRNA; EIciRNA, exonic-intronic circRNA; EMT, epithelial-to-mesenchymal transition; HCC, hepatocellular carcinoma; HIF1AN, hypoxia-inducible factor asparagine hydroxylase; ICMs, ntron complementary sequences; PBMCs, peripheral blood mononuclear cells; pre-mRNA, precursor messenger RNA; PI3K, phosphoinositide 3-kinase; QKI, quaking; RBP, RNAbinding protein; RCMs, reverse complementary matches; ROC, receiver operating characteristic; TAM, tumor-associatedmacrophage; TILs, tumor-infiltrating lymphocytes.
} 
diagnostic markers, leading to the great majority of patients are diagnosed with advanced or metastatic HCC. For these patients, the efficacy of local radiotherapy or chemotherapy is unsatisfactory, and there is little chance of curing by surgery. Thus, it is urgent to identify effective and sensitive diagnostic and prognostic markers, and to develop new treatment strategies for patients with HCC.

In recent years, a novel class of RNA molecule, circular RNA (circRNA), has received increasing attention due to its essential role in various diseases $(2,3)$, including human malignant tumors (4-7). CircRNA is formed by covalent closure of the $5^{\prime}$ and $3^{\prime}$ ends of the precursor messenger RNA (pre-mRNA) through back splicing (8). It contains a closed-loop structure that makes it resistant to digestion and cleavage of exonucleases, thus being highly stable in tissues and cells $(9,10)$. Additionally, most circRNAs exhibit high tissue- and cell-specific expression patterns and high conservation among species $(11,12)$. On the basis of these characteristics, circRNAs present great potential as indicators for disease diagnosis, progress monitoring and prognosis prediction in the clinic $(13,14)$.

Relying on the advancement of high-throughput sequencing and circRNA-related computational technologies, the biological function and clinical value of circRNA are gradually being explored. Emerging evidence suggests that circRNA involves in tumorigenesis. Li et al. (15) report that circACC1 promotes tumor cell growth by activating the AMPK complex under metabolic stress. Chen et al. (16) demonstrate that circRNA FECR1 promotes tumor deterioration by regulating DNA methylation and demethylating enzymes. Su et al. (17) indicate that circRNA cTFRC functions as a sponge of miR-107 to facilitate bladder cancer development. The critical role of circRNA in tumorigenesis makes it a prospective therapeutic target for cancer patients. Here, we summarized latest research on circRNA in HCC to outline the biological function, mechanism of action and clinical value of circRNA in HCC. The final goal of this review is to yielded more effective strategies for the diagnosis and prognosis prediction of HCC and to provide a basis for circRNA-based HCC molecular targeted therapy.

\section{BIOGENESIS OF CIRCRNA}

Up to now, the formation mechanism of circRNA has not been clearly understood. Majority of scientists consider that the biogenesis of circRNA differs from the linear mRNA: linear mRNA is produced by processing exons of pre-mRNA by canonical splice, while circRNA is formed by back-splicing and is regulated by cis-acting and trans-acting elements. Nowadays, the circularization mechanisms of circRNA have been suggested: (1) Intron pairing-driven circularization (Figure 1A); (2) RNAbinding protein (RBP)-driven circularization (Figure 1B); (3) Lariat-driven circularization (Figure 1C); (4) Lariat intronsdriven circularization (Figure 1D).

\section{Intron Pairing-Driven Circularization}

In this circularization model, the flanking introns of circRNA harbor reverse complementary sequences, resulting in base pairing, which shorten the distance between introns and connect the downstream $5^{\prime}$ splice site to the upstream $3^{\prime}$ splice site of pre-mRNA, thereby forming exonic circRNA (ecircRNA) or exonic-intronic circRNA (EIciRNA). Intron pairing-driven circularization depends on 30 40nt reverse complementary repeat elements, including reverse complementary matches (RCMs), intron complementary sequences (ICMs) and Alu elements. The foremost of these is Alu element, which is the main factor that facilitates intron pairing and mediates circularization in the mammalian genome, especially in the human genome $(18,19)$. Meanwhile, the competitive pairing between reserve complementary repeat elements leads to alternative splicing and thus promotes the diversity of circRNA.

Notably, not all introns can facilitate the back-splicing of premRNA and looping of exons. For instance, base-pairing may cause hairpin structure, resulting in failure to form circRNA (20).

\section{RBP-Driven Circularization}

By binding to the specific motif of an exon-flanking intron or forming dimers, RBP brings flanking introns together to regulate the looping of exons, thereby facilitating the formation of ecircRNA or EIciRNA. For instance, a previous study demonstrates that quaking (QKI), an RBP, regulate circRNA formation by binding to flanking introns and subsequently bringing downstream and upstream of the circRNA-forming exons together (21).

\section{Lariat-Driven Circularization}

In the lariat-driven circularization mechanism, the upstream $3^{\prime}$ splicing receptor of pre-mRNA covalently attaches to the downstream 5 ' splicing donor to form a lariat intermediate, which is composed of several exons and introns. Provided the introns are not completely spliced out but retained in the lariat, the lariat is likely to transform into ElciRNA. If all introns are removed, exons within lariat will form a connection by $3^{\prime}, 5^{\prime}$ phosphodiester bond, thereby generating ecircRNA.

\section{Lariat Introns-Driven Circularization}

After the process of pre-mRNA splicing, a majority of introns are degraded. However, the minority of introns which have a $7 \mathrm{nt}$ GU-rich motif in the $5^{\prime}$ splicing site and an 11 nt-C-rich motif in the $3^{\prime}$ branchpoint site may escape degradation by debranching enzyme, resulting in the generation of circular intronic RNA (ciRNA).

\section{BIOLOGICAL FUNCTION OF CIRCRNA}

Based on different formation mechanism and different components, circRNA is mainly divided into three subtypes: ecircRNA, EIciRNA, and ciRNA. EcircRNA consists of single or several exons and is primarily located in the cytoplasm. Previous evidence demonstrates that ecircRNA mainly functions in human tissues by acting as miRNA sponge (22) (Figure 2A), interacting with RBP (23) (Figure 2B) and translating protein (24) (Figure 2C). EIciRNA and ciRNA are intron-containing circRNAs, which are mainly located in the nucleus. They have 


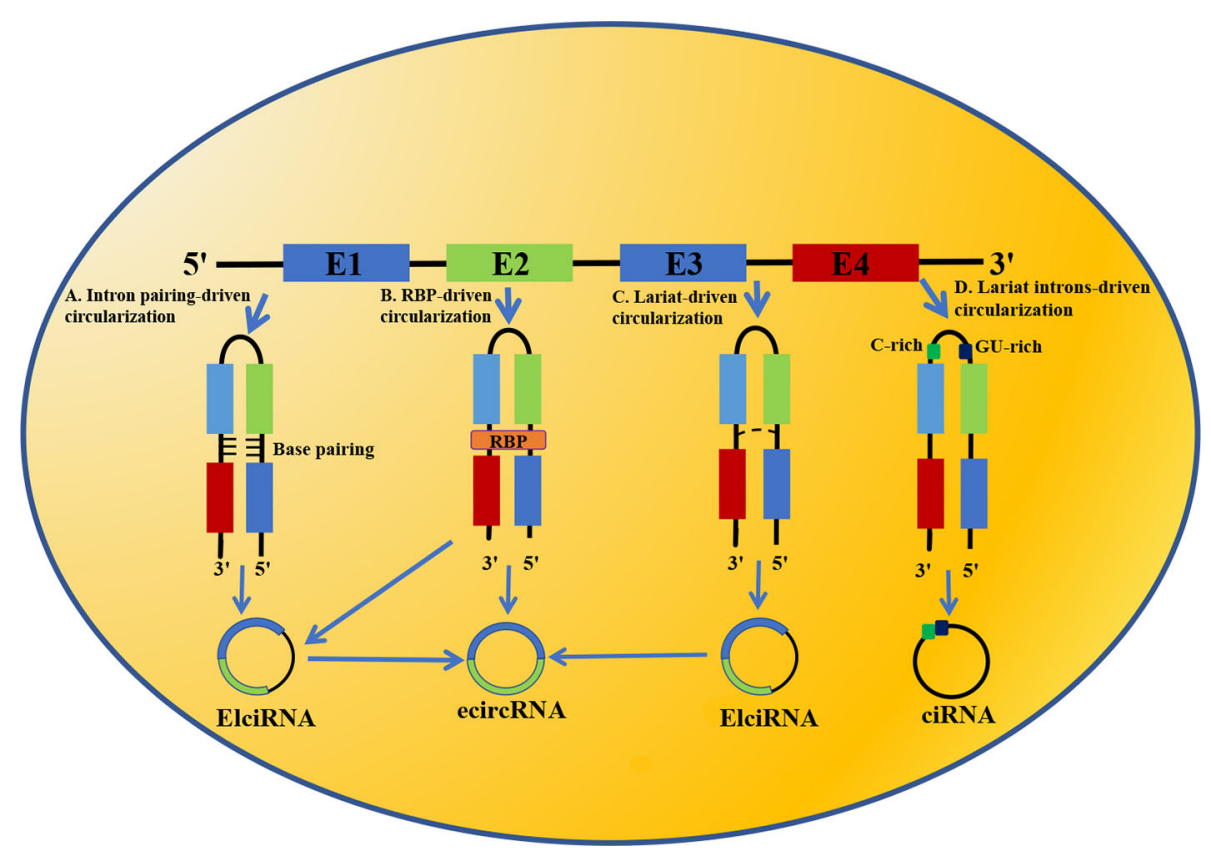

FIGURE 1 | Biogenesis of circRNA. (A) Intron pairing-driven circularization; (B) RNA binding protein (RBP)-driven circularization; (C) Lariat-driven circularization; (D) Lariat introns-driven circularization.

the potential to compete with linear mRNA splicing (25) (Figure 2D) and to regulate the transcription of parental genes $(26,27)$ (Figure 2E). Nowadays, most studies focus on the regulatory role of circRNA as a miRNA sponge in HCC. Further in-depth studies are needed to explore other mechanism by which circRNA affects HCC development.

\section{ROLE OF CIRCRNA IN HCC}

\section{CircRNA Promotes Tumor Progression in HCC}

In the long-term study of circRNA in HCC, researchers have found that some circRNAs promote the progression of HCC (Table 1 and Figure 3). Zhang et al. (28) demonstrate that circ_101141 accelerates the progression of HCC by sponging miR-1297 and regulating ROCK1 expression. He et al. (29) show that hsa_circ_0000517 is upregulated in HCC, and silencing hsa_circ_0000517 arrests HCC development by mediating the miR-326/SMAD6 axis. Cao et al. (30) find that silencing circRNA-103809 in HCC cells inhibits cell proliferation, migration, invasion, and epithelial-to-mesenchymal transition (EMT). Circ_0091579 accelerates HCC cell proliferation, migration, invasion, and glycolysis by targeting the miR-4905p/CASC3 axis (31). Circ-DENND4C, an onco-circRNA in breast cancer, is overexpression in HCC and increase the expression of TCF4 to activate the $\mathrm{Wnt} / \beta$-catenin signaling cascade by sponging miR-195-5p (32). Hsa_circ_0084922, also known as circ_KIAA1429, is upregulated in HCC, and facilitates
HCC advancement by upregulating Zeb1 in a m6A-YTHDF3 dependent manner (33). Hsa_circ_0026134 is overexpressed in HCC and promotes HCC growth and metastasis by sponging miR-127-5p to upregulate TRIM25 and IGF2BP3 (34). Sun et al. (35) demonstrate that circ_0005394 facilitates HCC growth via mediating the miR-507/E2F3 and miR-515-5p/CXCL6 axes. Jin et al. (36) find that hsa_circ_0101145 is overexpressed in HCC and silencing hsa_circ_0101145 inhibits the deterioration of HCC by targeting the miR-548c-3p/LAMC2 axis. Circ0003998, upregulated in portal vein tumor thrombus HCC tissues, is identified to promote HCC EMT by acting as miR-143-3p sponge to promote an EMT-related stimulator, FOSL2. Additionally, circ0003998 can bind with PCBP1 to enhance the expression of an EMT-related gene, CD44v6 (37). A study conducted by Huang et al. (38) shows that circMET is an onco-circRNA that promotes HCC evolvement and induces immune suppression by the Snail/DPP4/CXCL10 axis. Hu et al. (39) carry out a circRNA sequencing analysis on 15 samples from HCC patients with postoperative lung metastasis and 15 without metastasis or recurrence and determine circASAP1 as a metastasis-related circRNA in HCC. Mechanism analysis shows that circASAP1 accelerate HCC metastasis by targeting the miR-326/miR-532-5p-MAPK1 axis. Additionally, circASAP1 regulates tumor-associatedmacrophage (TAM) infiltration by mediating the miR-326/ miR-532-5p-CSF-1 axis. CircHIPK3 is overexpressed in HCC tissues, and knockdown of circHIPK3 decreases cell proliferation and migration by targeting miR-124 to regulate the expression of AQP3 (40). Huang et al. (41) find that circRNA-100338 


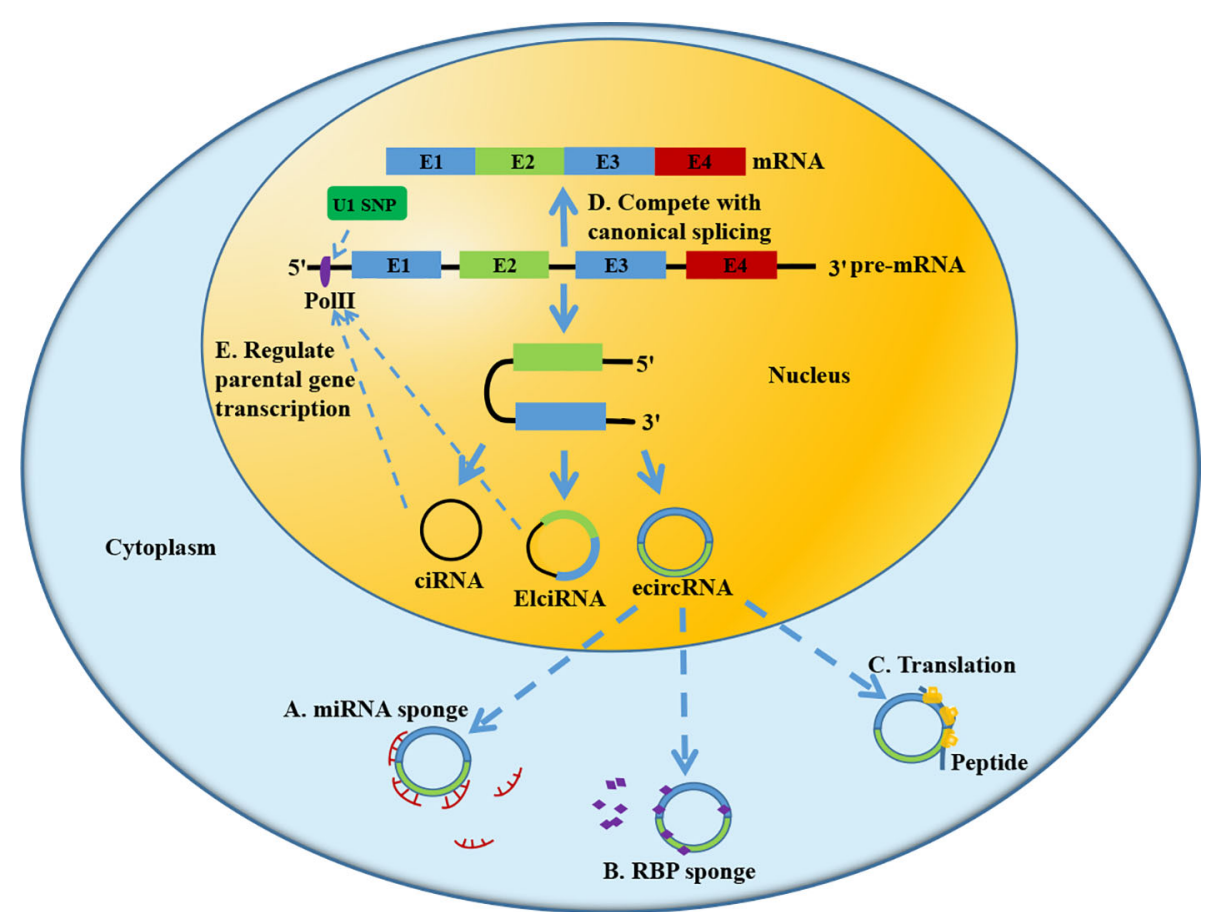

FIGURE 2 | Biological function of circRNA. (A) Acting as miRNA sponge; (B) Combining with RNA binding protein (RBP); (C) Translating protein; (D) Competing with splicing of pre-mRNA. (E) Regulating transcription of parental gene.

is overexpressed in HCC tissues and enhances HCC proliferation and migration by modulating the miR-141-3p/RHEB axis to activate mTOR signaling pathway. Also, circRNA-100338 is upregulated in both highly metastatic HCC cells and their secreted exosomes, and overexpressed or silencing exosomal circRNA-100338 remarkably promotes or reduces HCC invasion, indicating that circRNA-100338 is a metastasisrelated circRNA in HCC (42). Circ-BIRC6 is upregulated in HCC tissues and HCC cells. Overexpression of circ-BIRC6 enhances HCC cell proliferation, migration and invasion, and inhibits HCC cell apoptosis by targeting the miR-3918/Bcl2 axis (43). A study conducted by Wei et al. (44) shows that circ-CDYL is upregulated in early HCC and contributes to the chemoresistance and tumorigenic properties of HCC. Therapies combining circ-CDYL interference and traditional enzyme inhibitors targeting phosphoinositide 3-kinase (PI3K) and hypoxia-inducible factor asparagine hydroxylase (HIF1AN) effectively inhibit tumor growth in HCC. Zhang et al. (45) report that the exosome circ-DB secreted by adipocytes promotes HCC cell proliferation and reduces DNA damage by reducing miR-34a and inducing the USP7/Cyclin A2 signaling cascade. Gong et al. (46) show that Circ-ZEB1.33 facilitates HCC cell proliferation by promoting cells into S phase. Guo et al. (47) find that circ-ZNF652 is upregulated in HCC. Depletion of circZNF652 remarkably inhibits the migration and invasion of HCC cells in vitro, and suppresses HCC metastasis in vivo by binding to miR-203 and miR-502-5p to promote their common gene Snail, which is an important transcription factor that activates
EMT. Liang et al. (48) reveal that $\operatorname{circ} \beta$-catenin promotes HCC development by translation of a novel 370 -amino acid $\beta$-catenin isoform that can stabilize $\beta$-catenin and subsequent activate of the Wnt pathway. CircDYNC1H1 has been reported to sponge miR-140-5p, and silencing circDYNC1H inhibits the proliferation and migration of HCC cells by down-regulating SULT2B1 (49). CircFBLIM1 is upregulated in HCC. Knockdown of circFBLIM1 suppresses the proliferation and invasion of HCC cells and promotes their apoptosis by modulating FBLIM1 via binding to miR-346 (50). Li et al. (51) demonstrate that circMAT2B facilitates glycolysis and tumor growth in HCC by regulating the miR-338-3p/PKM2 axis under hypoxia. Wang et al. (52) report that circPTGR1, which contains three isoforms of hsa_circ_0003731, hsa_circ_0008043 and has_circ_0088030, is upregulated in serum exosomes of HCC patients. Cell migration and invasion are significantly inhibited in HCC cells with exosomes from the circPTGR1-knockdown cells. The authors further clarify that circPTGR1 promotes HCC growth via the miR-449a-MET pathway. Meng et al. (53) demonstrate that circ-10720 is regulated by a transcription factor Twist. Overexpressed circ-10720 in HCC cells promotes cell proliferation, migration and invasion, and knockdown of circ10720 in HCC cells inhibits cell migration and invasion. Liu et al. (54) report that knockdown of circ001569 significantly inhibits the growth, migration and invasion of Huh7 cells. Li et al. (55) find that circRNA101368 is overexpressed in HCC. Knockdown of circRNA101368 reduces HCC cell migration by regulating the miR-200a/HMGB1 axis. Study on hsa_circ_101280 shows that 


\begin{tabular}{|c|c|c|c|c|c|}
\hline CircRNA & Expression change & Experiment & Cell line & Biological function & Regulatory axis \\
\hline Circ_101141 & Upregulated in $\mathrm{HCC}$ & In vitro and in vivo & Hep3B, Huh7 & Proliferation (+), invasion (+), migration (+) & Circ_101141/miR-1297/ROCK1 \\
\hline Hsa_circ_0000517 & Upregulated in $\mathrm{HCC}$ & In vitro and in vivo & HCCLM3, Huh7 & $\begin{array}{l}\text { Proliferation }(+) \text {, colony formation }(+), \\
\text { migration }(+) \text {, invasion }(+)\end{array}$ & Hsa_circ_0000517/miR-326/SMAD6 \\
\hline CircRNA-103809 & Upregulated in $\mathrm{HCC}$ & In vitro & Huh7, HepG2 & $\begin{array}{l}\text { Proliferation (+), migration }(+) \text {, invasion }(+), \\
\text { EMT }(+)\end{array}$ & CircRNA-103809/miR-1270/PLAGL2 \\
\hline Circ_0091579 & Upregulated in $\mathrm{HCC}$ & In vitro & HCCLM3, Huh7 & $\begin{array}{l}\text { Proliferation (+), invasion (+), migration (+), } \\
\text { glycolysis }(+)\end{array}$ & Circ_0091579/miR-490-5p/CASC3 \\
\hline Circ-DENND4C & Upregulated in $\mathrm{HCC}$ & In vitro and in vivo & Huh7, HepG2 & $\begin{array}{l}\text { Proliferation (+), stemness }(+) \text {, invasion }(+), \\
\text { apoptosis }(-)\end{array}$ & Circ-DENND4C/miR-195-5p/TCF4/Wnt/ $\beta$-catenin signaling pathway \\
\hline Circ_KIAA1429 & Upregulated in $\mathrm{HCC}$ & In vitro and in vivo & HepG2, Bel7404 & Migration (+), invasion (+), EMT (+) & Circ_KIAA1429/m6AYYTHDF3/Zeb1 \\
\hline Hsa_circ_0026134 & Upregulated in $\mathrm{HCC}$ & In vitro and in vivo & HepaRG, HepG2 & Proliferation (+), invasion (+) & Hsa_circ_0026134/miR-127-5p/TRIM25/IGF2BP3 \\
\hline Circ_0005394 & Upregulated in HCC & In vitro & Huh7, HepG2 & Proliferation (+), migration (+), EMT (+) & Circ_0005394/miR-507/E2F3, circ_0005394/miR-515-5p/CXCL6 \\
\hline Hsa_circ_0101145 & Upregulated in $\mathrm{HCC}$ & In vitro and in vivo & Huh7, HepG2 & $\begin{array}{l}\text { Proliferation (+), invasion (+), migration }(+) \text {, } \\
\text { apoptosis }(-)\end{array}$ & Hsa_circ_0101145/miR-548c-3p/LAMC2 \\
\hline Circ0003998 & Upregulated in $\mathrm{HCC}$ & In vitro and in vivo & HepG2, MHCC97H & EMT $(+)$ & Circ0003998/mir-143-3p/FOSL2, circ0003998/PCBP1/CD44v6 \\
\hline CircMET & Upregulated in $\mathrm{HCC}$ & In vitro and in vivo & HCCLM3, HepG2 & $\begin{array}{l}\text { Migration (+), invasion (+), EMT (+), } \\
\text { immunosuppression }(+)\end{array}$ & CircMET/miR-30-5p/snail/DPP4/CXCL10 \\
\hline CircASAP1 & $\begin{array}{l}\text { Upregulated in } \\
\text { metastatic HCC }\end{array}$ & In vitro and in vivo & HepG2, MHCC97H & $\begin{array}{l}\text { Proliferation }(+) \text {, colony formation }(+) \text {, } \\
\text { migration }(+) \text {, invasion }(+) \text {, TAM infiltration } \\
(+)\end{array}$ & $\begin{array}{l}\text { CircASAP1/miR-326/miR-532-5p-MAPK1, circASAP1/miR-326/miR- } \\
\text { 532-5p-CSF-1 }\end{array}$ \\
\hline CircHIPK3 & Upregulated in HCC & In vitro and in vivo & Huh7, MHCC97H & Proliferation (+), metastasis (+) & CircHIPK3/miR-124/AQP3 \\
\hline CircRNA-100338 & Upregulated in HCC & In vitro & BEL7402, Нер3В & Proliferation $(+)$, metastasis $(+)$ & CircRNA-100338/miR-141-3p/RHEB/mTOR signaling pathway \\
\hline CircRNA-100338 & $\begin{array}{l}\text { Upregulated in } \\
\text { metastatic } \mathrm{HCC}\end{array}$ & In vitro and in vivo & Нер3В, МHCC97H & Invasion (+) & \\
\hline Circ-BIRC6 & Upregulated in $\mathrm{HCC}$ & In vitro and in vivo & HepG2, Bel7402 & $\begin{array}{l}\text { Proliferation (+), invasion (+), migration (+), } \\
\text { apoptosis }(-)\end{array}$ & Circ-BIRC6/miR-3918/Bcl2 \\
\hline Circ-CDYL & Upregulated in $\mathrm{HCC}$ & In vitro and in vivo & HCCLM3, SMMC7721 & Proliferation $(+)$ & $\begin{array}{l}\text { Cir-CDYL/miR-892a/HDGF/PIK3-AKT-mTORC1/B-catenin signaling } \\
\text { pathway, circ-CDYL/miR-328-3p/HIFIAN/NOTCH2 signaling pathway }\end{array}$ \\
\hline Circ-DB & Upregulated in $\mathrm{HCC}$ & In vitro & HepG2, Нера1-6 & Proliferation (+), migration (+) & Circ-DB/miR-34a/USP7/Cyclin A2 \\
\hline Circ-ZEB1.33 & Upregulated in HCC & In vitro & Huh7 & Proliferation (+) & Circ-ZEB1.33/miR-200a-3p/CDK6 \\
\hline Circ-ZNF652 & Upregulated in HCC & In vivo & HCCLM3, SK-HEP-1 & Migration (+), invasion (+), EMT (+) & Circ-ZNF652/miR-203/miR-502-5p/Snail \\
\hline Circ $\beta$-catenin & Upregulated in $\mathrm{HCC}$ & In vitro & Huh7, PLC-REF-5 & Proliferation (+), invasion (+) & Circ $\beta$-catenin $/ G S K 3 \beta / \beta$-catenin $/$ Wnt signaling pathway \\
\hline CircDYNC1H1 & Upregulated in $\mathrm{HCC}$ & In vitro & $\begin{array}{l}\text { SMMC7721, BEL- } \\
7402\end{array}$ & Proliferation $(+)$, migration $(+)$ & CircDYNC1H1/miR-140-5p/SULT2B1 \\
\hline CircFBLIM1 & Upregulated in HCC & In vitro and in vivo & $\begin{array}{l}\text { HepG2, BEL7402, } \\
\text { MHCC97H }\end{array}$ & Proliferation (+), invasion (+), apoptosis (-) & CircFBLIM1/miR-346/FBIM1 \\
\hline CircMAT2B & Upregulated in $\mathrm{HCC}$ & In vitro and in vivo & Huh7, HepG2 & Proliferation (+), migration (+), invasion (+) & CircMAT2B/miR-338-3p/PKM2 \\
\hline CircPTGR1 & Upregulated in $\mathrm{HCC}$ & In vitro and in vivo & HepG2, MHCC97L & Migration (+), invasion (+) & CircPTGR1/miR449a/MET \\
\hline Circ-10720 & $\begin{array}{l}\text { Upregulated in } \\
\text { metastatic HCC }\end{array}$ & In vitro and in vivo & $\begin{array}{l}\text { PLC-REF-5, } \\
\text { SMMC7721 }\end{array}$ & $\begin{array}{l}\text { Proliferation (+), migration (+), invasion (+), } \\
\text { EMT (+) }\end{array}$ & Twistl/Circ-10720/miR-490-5p/Nimentin \\
\hline Circ001569 & Upregulated in $\mathrm{HCC}$ & In vitro and in vivo & Huh7, HepG2 & Proliferation (+), migration (+), invasion (+) & Circ_001569/miR-411-5p/miR-432-5p \\
\hline CircRNA101368 & Upregulated in $\mathrm{HCC}$ & In vitro & HepG2, HCCLM3 & Migration (+) & CircRNA 101368/miR-200a/HMGB1 \\
\hline Hsa_Circ_101280 & Upregulated in HCC & In vitro and in vivo & HepG2, SNU-398 & Proliferation (+), apoptosis (-) & Hsa_Circ_101280/miR-375/JAK2 \\
\hline Has_circ_0016788 & Upregulated in $\mathrm{HCC}$ & In vitro and in vivo & Hep3B, Huh7 & Proliferation (+), invasion (+), apoptosis (-) & Hsa_Circ_0016788/miR-486/CDK4 \\
\hline Hsa_circ_0103809 & Upregulated in $\mathrm{HCC}$ & In vitro & HepG2, Huh7 & Proliferation $(+)$, migration $(+)$, apoptosis $(-)$ & Hsa_Circ_0103809/miR-490-5p/SOX2 \\
\hline CircNFATC3 & Downregulated in HCC & In vitro and in vivo & HepG2, Huh7 & $\begin{array}{l}\text { Proliferation (-), invasion (-), migration (-), } \\
\text { apoptosis (+) }\end{array}$ & CircNFATC3/miR-5481/NFATC3 \\
\hline CircMTO1 & Downregulated in $\mathrm{HCC}$ & In vitro & НерG2, Нер3В & Proliferation (-), migration (-), apoptosis (+) & CircMTO1/miR-9-5p/NOX1 \\
\hline
\end{tabular}

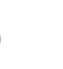




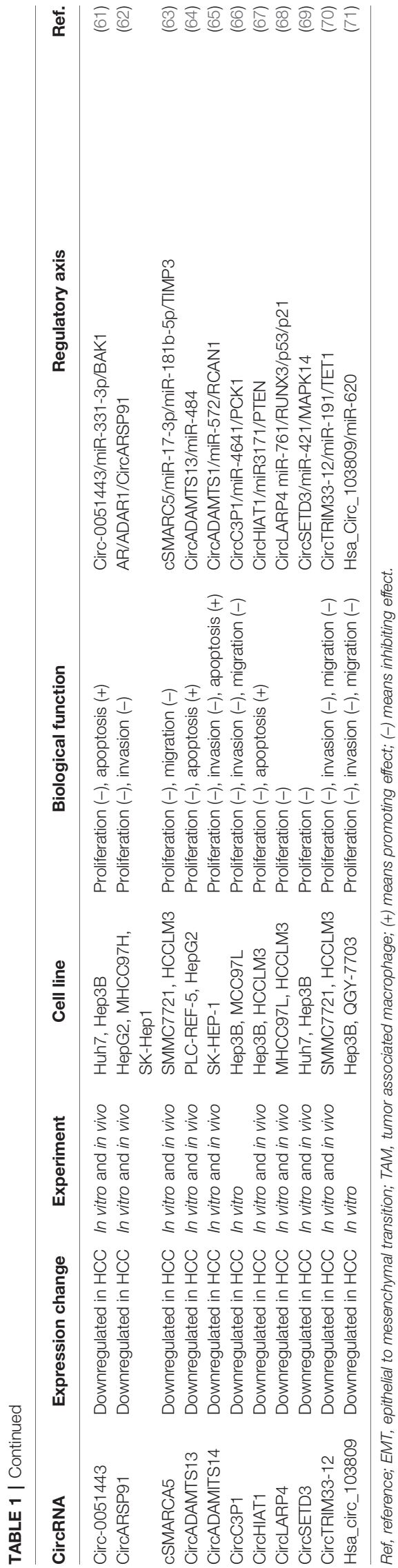

overexpressed hsa_circ_101280 promotes the proliferation and decreases the apoptosis of HCC cells in vitro by sponging miR375 to upregulate JAK2 (56). Guan et al. (57) indicate that silencing hsa_circ_0016788 suppresses the proliferation, invasion and promotes the apoptosis of HCC cells in vitro, and inhibits tumor growth in vivo. Cai et al. (58) find that knockdown of hsa_circ_0103809 inhibits the proliferation and migration and induces the apoptosis of HCC cells by targeting the miR-490-5p/ SOX2 axis. Our research group has been focusing on the role of circRNA in HCC. We have previously demonstrated that hsa_circ_0088364 and hsa_circ_0090049 are up-regulated in HCC cells (72). Hsa_circ_0088364 promotes HCC cells migration and inhibits HCC cells apoptosis. Has_circ_0090049 enhances cell proliferation and migration and suppresses cell apoptosis. Growing evidences indicate that some circRNAs, such as hsa_circ_00156 (73), hsa_circ_000224 (73), and hsa_circ_000520 (73), hsa_circ_0091579 (74), and hsa_circ_0128298 (75), are upregulated in HCC tissues or cells. But their biological functions or mechanisms of action still needed to be elucidated.

\section{CircRNA Inhibits Tumor Progression in HCC}

CircRNAs not only promote the progression of HCC but also delay HCC progression (Table $\mathbf{1}$ and Figure 3). CircNFATC3 is downregulated in HCC and impedes HCC development (59). Wang et al. (60) reveal that circMTO1 is low-expressed in HCC, and overexpression of circMTO1 inhibits the proliferation and migration and induces the apoptosis of HCC cells. Circ-0051443, a circRNA that is transported from normal cells to HCC cells via exosomes, suppresses HCC progression by sponging miR-331-3p to induce the expression of BAK1 (61). CircARSP91, a circRNA that is suppressed by AR in an ADAR1-dependent manner, functions as a tumor suppressor in HCC (62). Yu et al. (63) indicate that CSMARCA5 promotes the expression of TIMP3 (a well-known tumor suppressor) by binding to miR-17-3p and miR-181b-5p, and then slower the growth of HCC. Qiu et al. (64) perform an extensive analysis of circRNA profiles in $10 \mathrm{HCC}$ tumor tissues and paired nontumor tissues and discover that circADAMTS13 is remarkably downregulated in HCC tissues. Overexpression of circADAMTS13 in HCC cells inhibits cell proliferation and promotes cell apoptosis by sponging miR-484. Song et al. (65) reveal that circADAMITS14 induces HCC cell apoptosis and inhibits HCC cell proliferation and invasion by regulating miR-572/RCAN1. Zhong et al. (66) find that circC3P1 suppresses the growth and metastasis of HCC by sponging miR4641 to enhance PCK1 expression. CircH1AT1 is demonstrated to inhibit tumor growth in HCC in vivo and in vitro by targeting the miR-3171/PTEN axis (67). CircLARP4 is found to suppress tumor growth in HCC by mediating the miR-76/RUNX3/p53/ p21 signaling pathway (68). Xu et al. (69) demonstrate that circSETD3 restrain the proliferation of HCC cells by blocking HCC cells in the G1/S phase. Zhang et al. (70) show that the decreased expression of circTRIM33-12 promotes tumor proliferation, migration, invasion, and immune evasion by binding to miR-191 to upregulate TET1 expression. It is reported that hsa_circ_103809 is significantly downregulated in 


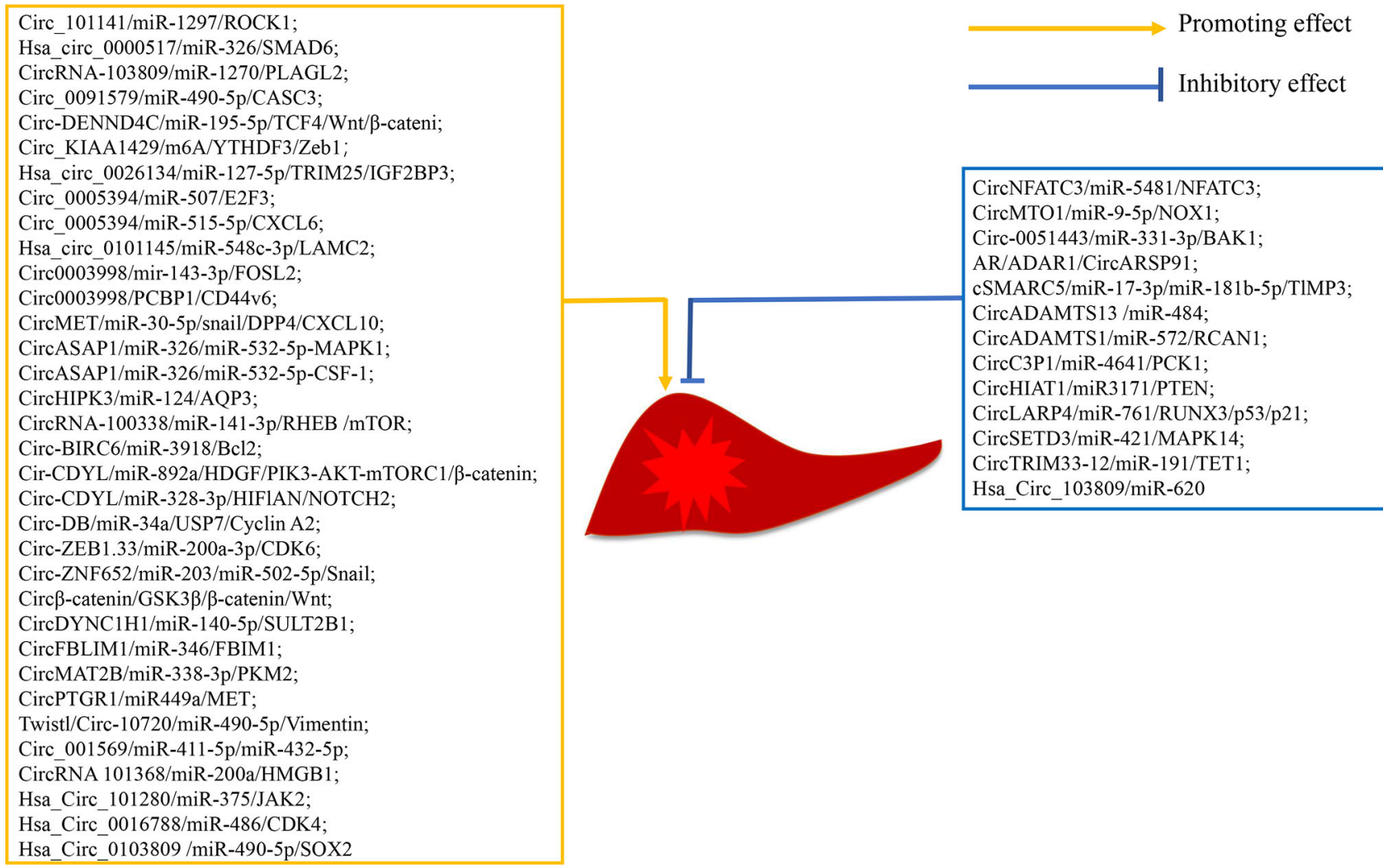

FIGURE 3 | Mechanism of action of circRNAs in hepatocellular carcinoma.

HCC tissues and cell lines and acts as a miRNA sponge of miR620 to inhibit the proliferation and invasion of HCC cells (71).

So far, people's cognition and understanding of the correlation between circRNA and HCC is just the tip of an iceberg, and many unknown fields need to be studied. For example, some studies have observed that circRNA can induce DNA methylation or demethylation of $\mathrm{CpG}$ island of gene promoters, leading to the activation or inactivation of key genes or signaling pathways, thereby affecting disease development $(16,76)$. A recent study reveals that circRNA can undergo N6-methyladenosine (m6A) modification, which promotes protein translation (77). However, the relationships between circRNA and DNA methylation and m6A modification have not been elucidated in HCC. Whether circRNA can be modified by m6A or affect the methylation of other genes in HCC deserves further study.

\section{CLINICAL SIGNIFICANCE OF CIRCRNA IN HCC}

\section{Application of circRNA in the Diagnosis of HCC}

Increasing studies reveal that circRNA shows good performance in differentiating HCC patients from normal controls, indicating that it is expected to be a biomarker for HCC diagnosis.
Wu et al. (78) reveal that circ_0009582, circ_0037120 and circ_0140117 are increased in HBV-related HCC samples comparing with either chronic hepatitis or healthy controls. Combination of the three circRNAs and AFP can be employed to predict the occurrence HCC with $\mathrm{HBV}$ infection. Cheng et al. (79) report that circ_0016788 is upregulated in HCC tissues and it shows an excellent performance in distinguishing HCC samples from normal controls (area under the curve (AUC): 0.913). Circ-0051443 is mainly packaged in exosomes in HCC (61). The receiver operating characteristic (ROC) curve shows that the exosomal circ-0051443 can distinguish HCC patients from normal controls (ACU: 0.8089). CircRNA_104075 is highly expressed in HCC and shows an excellent diagnostic value in HCC patients, with an AUC value of 0.973 (80). Guan et al. (57) demonstrate a high diagnostic value of hsa_circ_0016788 in HCC (AUC=0.851).

In addition to in HCC tissues, abnormally expressed circRNA can also be detected in peripheral blood of patients with HCC, which provides a more convenient idea for the development of the diagnostic value of circRNA. Sun et al. (81) report that hsa_circ_0004001, hsa_circ_0004123 and hsa_circ_0075792 are upregulated in the blood samples of HCC. ROC analysis shows that the AUC value of the combination of the three circRNAs is 0.89 , indicating that the combination of the three circRNAs could be a potential diagnostic biomarker in HCC. Lei et al. (82) conducted an RNA-seq analysis to identify differently expressed 
circRNAs in peripheral blood mononuclear cells (PBMCs) of HCC patients and normal controls. They find that circ_0000798 can distinguish HCC patients from healthy controls, suggesting that it may be a promising biomarker that can be detected in blood for the diagnosis of HCC. The above evidences indicate that circRNAs are expected to be used clinically for HCC diagnosis.

\section{Application of circRNA in the Prognosis of HCC}

CircRNA is highly tissue-specific and more stable than linear mRNA, making it a promising prognostic marker for HCC. Circ_0005394 is upregulated in HCC, and overexpression of circ_0005394 is linked with larger tumor size, more advanced TNM stages, and poorer survival outcome for HCC patients (35). An onco-circRNA, circMET, is identified to be an independent predictor of overall survival and postoperative recurrence in HCC patients (38). Circ_0000267 is reported to be closely related to the clinical severity and worse survival outcome of patients with HCC (83). Chen et al. (75) show that the upregulated has_circ_0128298 is an independent factor for the prognosis prediction in patients with HCC. An oncogenic circRNA, circSLC3A2, is overexpression in HCC tissues and is associated with poor survival outcome in HCC patients (84). CircSETD3 is downregulated in HCC tissues, low expression of circSETD3 indicates shorter survival time in patients with HCC (69). The previous report shows that tumor patients with high tumor-infiltrating lymphocytes (TILs) have favorable survival outcome. Weng et al. (85) perform a microarray analysis by using plasma of HCC patients with high- and low-TILs and find that hsa_circ_0064428 is downregulated in patients with high TILs and is negatively related to overall survival, indicating that hsa_circ_0064428 is a promising immune-associated prognostic marker for patients with HCC.

A biomarker that can be detected from peripheral blood and other body fluids by non-invasive methods has better clinical application value. A study conducted by Gong et al. (46) indicates that circ-ZEB1.33 is upregulated in HCC tissues and predicts unfavorable prognosis in HCC patients. In addition, the authors demonstrate that circ-ZEB1.33 can also be detected in patients' serum and that its expression in the serum of HCC patients is remarkably higher than that in healthy control. More importantly, the high expression of circ-ZEB1.33 in the serum of HCC patients indicates a shorter survival time. These findings suggest that circ-ZEB1.33 expression can be detected clinically by noninvasive methods to assess the prognosis of HCC patients. CircRNA_101237 is identified to be upregulated in the serum of HCC patients as compared with that in normal controls, and high expression of serum circRNA_101237 predicts unfavorable clinical outcome, indicating that serum circRNA_101237 can be a promising biomarker for prognosis prediction in HCC patients (86).

\section{Application of circRNA in the Treatment of HCC}

Although treatment methods have been developed, the therapeutic effect of HCC is yet not optimistic. In the past few decades, the exploration of circRNA in HCC provides a breakthrough for future HCC treatment.

On the one hand, circRNA regulates certain signaling pathways, such as mTOR signaling pathway (41), $\beta$-catenin signaling pathway (44), NOTCH2 signaling pathway (44), Wnt signaling pathway (48), and p53/p21 signaling pathway (68), which play critical roles in the progression of HCC. Changing circRNA expression leads to the activation or inactivation of these signaling pathways, thereby affecting the progression of HCC. On the other hand, circRNA activates or inactivates some oncogenes and tumor suppressor genes, affecting the evolvement of HCC. For example, circ-BIRC6 enhances the expression of Bcl2, a well-known oncogene, to promote HCC development (43). All these evidences indicate that circRNA plays a key role in the development of HCC. Increasing or inhibiting the expression of circRNA in HCC patients may reverse the progress of HCC. However, the technique of specifically altering the expression of a specified circRNA in human body still needs further study. Increasing studies show that nanomaterials are promising drug carriers for cancer therapy. Nano-based delivery of circRNA overexpression or RNA interference molecules into tumor tissues of HCC patients is expected to be used for molecular targeted therapy of HCC patients.

\section{Relationship Between circRNA and Chemotherapy Resistance in HCC}

It is well known that drug resistance occurs in the chemotherapy of most cancers at a later stage, leading to unsatisfactory therapeutic effects. Recent studies have focused on the involvement of dysregulated circRNA in HCC drug resistance (Figure 4). Huang et al. (87) show that circFoxo3 is overexpressed in Adriamycin-resistant HCC tissues and cell lines and circFoxo3 may induce Adriamycin resistance via the miR-199a$5 \mathrm{p} / \mathrm{ABCC} 1$ axis. CircUHRF1 is secreted by HCC cells and transports to plasma by exosomes, and a high level of exosomal circUHRF1 contributes to natural killer cell exhaustion and drives resistance to anti-PD1 immunotherapy in HCC (88). Zhou et al. (86) reveal that the expression of circRNA_101237 in the serum of cisplatin-resistant HCC patients and cisplatinresistant Huh7 cells is elevated, indicating that circRNA_101237 contributes to cisplatin-resistant in patients with HCC. A study conducted by Chen et al. (89) shows that circ_0003418 is downregulated in HCC. Knockdown of circ_0003418 enhanced cisplatin resistance of HCC cells. Interestingly, the authors also find that silencing circ_0003418 results in the activation of the Wnt $/ \beta$-catenin signaling cascade, and the promotion of circ 0003418 on the sensitivity of HCC cells to cisplatin is reversed after inhibiting $\mathrm{Wnt} / \beta$-catenin pathway.

The above evidences show that circRNA is expected to be a prospective biomarker for the diagnosis and prognosis assessment of cancer patients. However, there are still several problems to be settled in the clinical application of circRNA as a biomarker. For example, the non-invasive examination has more excellent clinical application value, while most previous studies focus on the expression of circRNA in HCC tissues and only a 


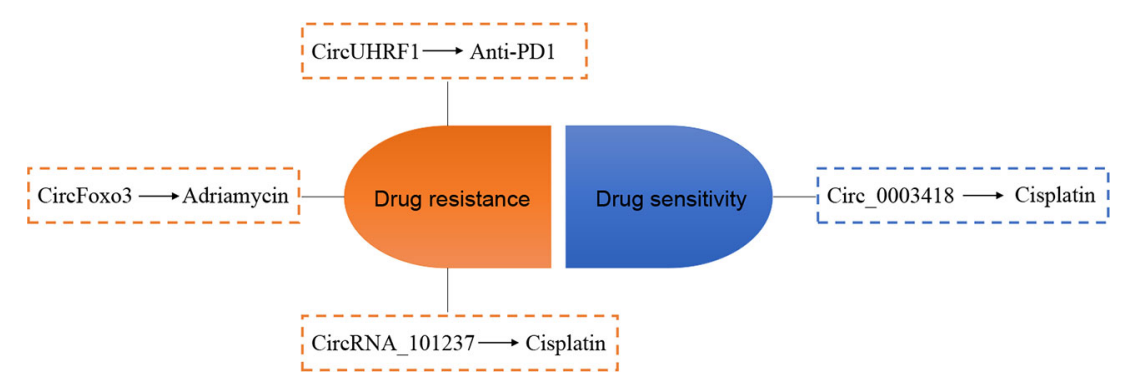

FIGURE 4 | Relationship between circRNA and chemotherapy resistance in hepatocellular carcinoma (HCC). The arrows indicate promotion effect.

few studies have focused on the expression of circRNA in peripheral blood, urine, saliva and other body fluids. In addition, circRNA has been demonstrated to be related to the chemotherapy resistance of HCC patients, so how to improve the sensitivity of traditional chemotherapeutics and immunotherapy by targeting circRNA clinically is worth attention. Therefore, we need to do more in-depth studies to determine the relationship between circRNA and HCC, exploring more accurate and straightforward detection methods, selecting more stable and suitable circRNA as a diagnostic and prognostic biomarker or treatment target in patients with HCC.

\section{CONCLUSION AND PERSPECTIVE}

Growing studies have shown that circRNA is differently expressed in various human malignant tumors. Differentially expressed circRNA is involved in tumor development by functioning as miRNA sponges, interacting with RBPs, translating proteins, competing with linear mRNA splicing of pre-mRNA and regulating parental genes. In this study, we comprehensively tracked the latest progress of circRNA in the pathogenesis of HCC. The circRNA-related studies have brought us many surprising findings suggesting that circRNA participates in the pathobiology of HCC. However, there are some open questions. Firstly, thousands of circRNAs in HCC have been detected and identified by RNA-seq, but only a small amount of functional circRNA has been characterized in HCC. It is necessary to analyze the function of more circRNAs and to understand the cell location, transportation, and degradation of circRNAs, which will

\section{REFERENCES}

1. Bray F, Ferlay J, Soerjomataram I, Siegel RL, Torre LA, Jemal A. Global cancer statistics 2018: GLOBOCAN estimates of incidence and mortality worldwide for 36 cancers in 185 countries. CA Cancer J Clin (2018) 68:394-424. doi: $10.3322 /$ caac. 21492

2. Nahand JS, Jamshidi S, Hamblin MR, Mahjoubin-Tehran M, Vosough M, Jamali M, et al. Circular RNAs: New Epigenetic Signatures in Viral Infections. Front Microbiol (2020) 11:1853. doi: 10.3389/fmicb. 2020.01853 enrich our knowledge of the complex regulatory networks involved in hepatocarcinogenesis. Secondly, circRNA has been reported to be a promising diagnostic and prognostic marker of HCC, but most of the previous studies have focused on circRNAs those expressed in HCC tissues. In fact, circRNAs those secreted in peripheral blood, exosomes, urine, and saliva are easier to be detected clinically. Therefore, more study is needed to mine circRNAs exist in body fluids to develop circRNA detection kits for HCC screening and prognosis monitoring. In conclusion, more studies are needed to disclose the relationship between circRNA and HCC to promote the clinical application of circRNA in HCC diagnosis, prognosis prediction, and therapy.

\section{AUTHOR CONTRIBUTIONS}

Study concept and design: DX and GC. Analysis and interpretation of data: DX, RH, and YD. Drafting of the manuscript: DX and HW. Critical revision of the manuscript for important intellectual content: DX, RH, and GC. Obtained funding: ZF and GC. Study supervision: HW and GC. All authors contributed to the article and approved the submitted version.

\section{FUNDING}

This study is supported by the Natural Science Foundation of Guangxi, China (2018GXNSFAA294025) and the Guangxi Degree and Postgraduate Education Reform and Development Research Projects, China (JGY2019050).

3. Abbaszadeh-Goudarzi K, Radbakhsh S, Pourhanifeh $\mathrm{MH}$, Khanbabaei H, Davoodvandi A, Fathizadeh H, et al. and Diabetes: Epigenetic Regulator with Diagnostic Role. Curr Mol Med (2020) 20:516-26. doi: 10.2174/ 1566524020666200129142106

4. Liu J, Li D, Luo H, Zhu X. Circular RNAs: The star molecules in cancer. Mol Aspects Med (2019) 70:141-52. doi: 10.1016/j.mam.2019. 10.006

5. Yin Y, Long J, He Q, Li Y, Liao Y, He P, et al. Emerging roles of circRNA in formation and progression of cancer. J Cancer (2019) 10:5015-21. doi: $10.7150 /$ jca.30828 
6. Shang BQ, Li ML, Quan HY, Hou PF, Li ZW, Chu SF, et al. Functional roles of circular RNAs during epithelial-to-mesenchymal transition. Mol Cancer (2019) 18:138. doi: 10.1186/s12943-019-1071-6

7. Borran S, Ahmadi G, Rezaei S, Anari MM, Modabberi M, Azarash Z, et al. Circular RNAs: New players in thyroid cancer. Pathol Res Pract (2020) 216:153217. doi: 10.1016/j.prp.2020.153217

8. Santer L, Bar C, Thum T. Circular RNAs: A Novel Class of Functional RNA Molecules with a Therapeutic Perspective. Mol Ther (2019) 27:1350-63. doi: 10.1016/j.ymthe.2019.07.001

9. Li Y, Ge YZ, Xu L, Jia R. Circular RNA ITCH: A novel tumor suppressor in multiple cancers. Life Sci (2020) 254:117176. doi: 10.1016/j.lfs.2019.117176

10. Li D, Li Z, Yang Y, Zeng X, Li Y, Du X, et al. Circular RNAs as biomarkers and therapeutic targets in environmental chemical exposure-related diseases. Environ Res (2020) 180:108825. doi: 10.1016/j.envres.2019.108825

11. Kristensen LS, Andersen MS, Stagsted LVW, Ebbesen KK, Hansen TB, Kjems J. The biogenesis, biology and characterization of circular RNAs. Nat Rev Genet (2019) 20:675-91. doi: 10.1038/s41576-019-0158-7

12. Cui X, Wang J, Guo Z, Li M, Li M, Liu S, et al. Emerging function and potential diagnostic value of circular RNAs in cancer. Mol Cancer (2018) 17:123. doi: 10.1186/s12943-018-0877-y

13. Meng S, Zhou H, Feng Z, Xu Z, Tang Y, Li P, et al. CircRNA: functions and properties of a novel potential biomarker for cancer. Mol Cancer (2017) 16:94. doi: 10.1186/s12943-017-0663-2

14. Naeli P, Pourhanifeh MH, Karimzadeh MR, Shabaninejad Z, Movahedpour A, Tarrahimofrad $\mathrm{H}$, et al. Circular RNAs and gastrointestinal cancers: Epigenetic regulators with a prognostic and therapeutic role. Crit Rev Oncol Hematol (2020) 145:102854. doi: 10.1016/j.critrevonc.2019.102854

15. Li Q, Wang Y, Wu S, Zhou Z, Ding X, Shi R, et al. CircACC1 Regulates Assembly and Activation of AMPK Complex under Metabolic Stress. Cell Metab (2019) 30:157-73.e7. doi: 10.1016/j.cmet.2019.05.009

16. Chen N, Zhao G, Yan X, Lv Z, Yin H, Zhang S, et al. A novel FLI1 exonic circular RNA promotes metastasis in breast cancer by coordinately regulating TET1 and DNMT1. Genome Biol (2018) 19:218. doi: 10.1186/s13059-018$1594-y$

17. Su H, Tao T, Yang Z, Kang X, Zhang X, Kang D, et al. Circular RNA cTFRC acts as the sponge of MicroRNA-107 to promote bladder carcinoma progression. Mol Cancer (2019) 18:27. doi: 10.1186/s12943-019-0951-0

18. Jeck WR, Sorrentino JA, Wang K, Slevin MK, Burd CE, Liu J, et al. Circular RNAs are abundant, conserved, and associated with ALU repeats. RNA (2013) 19:141-57. doi: 10.1261/rna.035667.112

19. Zhang XO, Wang HB, Zhang Y, Lu X, Chen LL, Yang L. Complementary sequence-mediated exon circularization. Cell (2014) 159:134-47. doi: 10.1016/ j.cell.2014.09.001

20. Liang D, Wilusz JE. Short intronic repeat sequences facilitate circular RNA production. Genes Dev (2014) 28:2233-47. doi: 10.1101/gad.251926.114

21. Conn SJ, Pillman KA, Toubia J, Conn VM, Salmanidis M, Phillips CA, et al. The RNA binding protein quaking regulates formation of circRNAs. Cell (2015) 160:1125-34. doi: 10.1016/j.cell.2015.02.014

22. Zhao ZJ, Shen J. Circular RNA participates in the carcinogenesis and the malignant behavior of cancer. RNA Biol (2017) 14:514-21. doi: 10.1080/ 15476286.2015 .1122162

23. Han J, Meng J, Chen S, Wang X, Yin S, Zhang Q, et al. YY1 Complex Promotes Quaking Expression via Super-Enhancer Binding during EMT of Hepatocellular Carcinoma. Cancer Res (2019) 79:1451-64. doi: 10.1158/00085472.CAN-18-2238

24. Pamudurti NR, Bartok O, Jens M, Ashwal-Fluss R, Stottmeister C, Ruhe L, et al. Translation of CircRNAs. Mol Cell (2017) 66:9-21.e7. doi: 10.1016/ j.molcel.2017.02.021

25. Ashwal-Fluss R, Meyer M, Pamudurti NR, Ivanov A, Bartok O, Hanan M, et al. circRNA biogenesis competes with pre-mRNA splicing. Mol Cell (2014) 56:55-66. doi: 10.1016/j.molcel.2014.08.019

26. Chen LL. The biogenesis and emerging roles of circular RNAs. Nat Rev Mol Cell Biol (2016) 17:205-11. doi: 10.1038/nrm.2015.32

27. Li Z, Huang C, Bao C, Chen L, Lin M, Wang X, et al. Exon-intron circular RNAs regulate transcription in the nucleus. Nat Struct Mol Biol (2015) 22:256-64. doi: 10.1038/nsmb.2959

28. Zhang T, Zhang L, Han D, Tursun K, Lu X. Circular RNA hsa_Circ_101141 as a Competing Endogenous RNA Facilitates Tumorigenesis of Hepatocellular
Carcinoma by Regulating miR-1297/ROCK1 Pathway. Cell Transplant (2020) 29:963689720948016. doi: 10.1177/0963689720948016

29. He S, Guo Z, Kang Q, Wang X, Han X. Circular RNA hsa_circ_0000517 modulates hepatocellular carcinoma advancement via the miR-326/SMAD6 axis. Cancer Cell Int (2020) 20:360. doi: 10.1186/s12935-020-01447-w

30. Cao Y, Tao Q, Kao X, Zhu X. Hsa-circRNA-103809 Promotes Hepatocellular Carcinoma Development via MicroRNA-1270/PLAG1 Like Zinc Finger 2 Axis. Dig Dis Sci (2020). doi: 10.1007/s10620-020-06416-x

31. Liu W, Yin C, Liu Y. Circular RNA circ_0091579 Promotes Hepatocellular Carcinoma Proliferation, Migration, Invasion, and Glycolysis Through miR490-5p/CASC3 Axis. Cancer Biother Radiopharm (2020). doi: 10.1089/ cbr.2019.3472

32. Liu X, Yang L, Jiang D, Lu W, Zhang Y. Circ-DENND4C up-regulates TCF4 expression to modulate hepatocellular carcinoma cell proliferation and apoptosis via activating Wnt/beta-catenin signal pathway. Cancer Cell Int (2020) 20:295. doi: 10.1186/s12935-020-01346-0

33. Wang M, Yang Y, Yang J, Yang J, Han S. circ_KIAA1429 accelerates hepatocellular carcinoma advancement through the mechanism of $\mathrm{m}(6) \mathrm{A}$ YTHDF3-Zeb1. Life Sci (2020) 257:118082. doi: 10.1016/j.lfs.2020.118082

34. Zhang W, Zhu L, Yang G, Zhou B, Wang J, Qu X, et al. Hsa_circ_0026134 expression promoted TRIM25- and IGF2BP3-mediated hepatocellular carcinoma cell proliferation and invasion via sponging miR-127-5p. Biosci Rep (2020) 40:BSR20191418. doi: 10.1042/BSR20191418

35. Sun C, Li G, Liu MA, Novel Circular RNA. circ_0005394, Predicts Unfavorable Prognosis and Contributes to Hepatocellular Carcinoma Progression by Regulating miR-507/E2F3 and miR-515-5p/CXCL6 Signaling Pathways. Onco Targets Ther (2020) 13:6171-80. doi: 10.2147/OTT.S256238

36. Jin J, Liu H, Jin M, Li W, Xu H, Wei F. Silencing of hsa_circ_0101145 reverses the epithelial-mesenchymal transition in hepatocellular carcinoma via regulation of the miR-548c-3p/LAMC2 axis. Aging (Albany NY) (2020) 12:11623-35. doi: 10.18632/aging.103324

37. Song LN, Qiao GL, Yu J, Yang CM, Chen Y, Deng ZF, et al. Hsa_circ_0003998 promotes epithelial to mesenchymal transition of hepatocellular carcinoma by sponging miR-143-3p and PCBP1. J Exp Clin Cancer Res (2020) 39:114. doi: 10.1186/s13046-020-01576-0

38. Huang XY, Zhang PF, Wei CY, Peng R, Lu JC, Gao C, et al. Circular RNA circMET drives immunosuppression and anti-PD1 therapy resistance in hepatocellular carcinoma via the miR-30-5p/snail/DPP4 axis. Mol Cancer (2020) 19:92. doi: 10.1186/s12943-020-01213-6

39. Hu ZQ, Zhou SL, Li J, Zhou ZJ, Wang PC, Xin HY, et al. Circular RNA Sequencing Identifies CircASAP1 as a Key Regulator in Hepatocellular Carcinoma Metastasis. Hepatology (2019) 72:906-22. doi: 10.1002/hep.31068

40. Chen G, Shi Y, Liu M, Sun J. circHIPK3 regulates cell proliferation and migration by sponging miR-124 and regulating AQP3 expression in hepatocellular carcinoma. Cell Death Dis (2018) 9:175. doi: 10.1038/s41419017-0204-3

41. Huang XY, Huang ZL, Zhang PB, Huang XY, Huang J, Wang HC, et al. CircRNA-100338 Is Associated With mTOR Signaling Pathway and Poor Prognosis in Hepatocellular Carcinoma. Front Oncol (2019) 9:392. doi: $10.3389 /$ fonc. 2019.00392

42. Huang XY, Huang ZL, Huang J, Xu B, Huang XY, Xu YH, et al. Exosomal circRNA-100338 promotes hepatocellular carcinoma metastasis via enhancing invasiveness and angiogenesis. J Exp Clin Cancer Res (2020) 39:20. doi: 10.1186/s13046-020-1529-9

43. Yang G, Wang X, Liu B, Lu Z, Xu Z, Xiu P, et al. circ-BIRC6, a circular RNA, promotes hepatocellular carcinoma progression by targeting the miR-3918/ Bcl2 axis. Cell Cycle (2019) 18:976-89. doi: 10.1080/15384101.2019.1601477

44. Wei Y, Chen X, Liang C, Ling Y, Yang X, Ye X, et al. A Noncoding Regulatory RNAs Network Driven by Circ-CDYL Acts Specifically in the Early Stages Hepatocellular Carcinoma. Hepatology (2020) 71:130-47. doi: 10.1002/ hep.30795

45. Zhang H, Deng T, Ge S, Liu Y, Bai M, Zhu K, et al. Exosome circRNA secreted from adipocytes promotes the growth of hepatocellular carcinoma by targeting deubiquitination-related USP7. Oncogene (2019) 38:2844-59. doi: 10.1038/s41388-018-0619-z

46. Gong Y, Mao J, Wu D, Wang X, Li L, Zhu L, et al. Circ-ZEB1.33 promotes the proliferation of human HCC by sponging miR-200a-3p and upregulating CDK6. Cancer Cell Int (2018) 18:116. doi: 10.1186/s12935-018-0602-3 
47. Guo J, Duan H, Li Y, Yang L, Yuan L. A novel circular RNA circ-ZNF652 promotes hepatocellular carcinoma metastasis through inducing snailmediated epithelial-mesenchymal transition by sponging miR-203/miR-5025p. Biochem Biophys Res Commun (2019) 513:812-9. doi: 10.1016/ j.bbrc.2019.03.214

48. Liang WC, Wong CW, Liang PP, Shi M, Cao Y, Rao ST, et al. Translation of the circular RNA circbeta-catenin promotes liver cancer cell growth through activation of the Wnt pathway. Genome Biol (2019) 20:84. doi: 10.1186/ s13059-019-1685-4

49. Wang ZY, Zhu Z, Wang HF, Qin B, Liu J, Yao XH, et al. Downregulation of circDYNC1H1 exhibits inhibitor effect on cell proliferation and migration in hepatocellular carcinoma through miR-140-5p. J Cell Physiol (2019) 234:17775-85. doi: 10.1002/jcp.28403

50. Bai N, Peng E, Qiu X, Lyu N, Zhang Z, Tao Y, et al. circFBLIM1 act as a ceRNA to promote hepatocellular cancer progression by sponging miR-346. J Exp Clin Cancer Res (2018) 37:172. doi: 10.1186/s13046-018-0838-8

51. Li Q, Pan X, Zhu D, Deng Z, Jiang R, Wang X. Circular RNA MAT2B Promotes Glycolysis and Malignancy of Hepatocellular Carcinoma Through the miR-338-3p/PKM2 Axis Under Hypoxic Stress. Hepatology (2019) 70:1298-316. doi: 10.1002/hep.30671

52. Wang G, Liu W, Zou Y, Wang G, Deng Y, Luo J, et al. Three isoforms of exosomal circPTGR1 promote hepatocellular carcinoma metastasis via the miR449a-MET pathway. EBioMedicine (2019) 40:432-45. doi: 10.1016/ j.ebiom.2018.12.062

53. Meng J, Chen S, Han JX, Qian B, Wang XR, Zhong WL, et al. Twist1 Regulates Vimentin through Cul2 Circular RNA to Promote EMT in Hepatocellular Carcinoma. Cancer Res (2018) 78:4150-62. doi: 10.1158/0008-5472.CAN-173009

54. Liu H, Xue L, Song C, Liu F, Jiang T, Yang X. Overexpression of circular RNA circ_001569 indicates poor prognosis in hepatocellular carcinoma and promotes cell growth and metastasis by sponging miR-411-5p and miR432-5p. Biochem Biophys Res Commun (2018) 503:2659-65. doi: 10.1016/ j.bbrc.2018.08.020

55. Li S, Gu H, Huang Y, Peng Q, Zhou R, Yi P, et al. Circular RNA 101368/miR200a axis modulates the migration of hepatocellular carcinoma through HMGB1/RAGE signaling. Cell Cycle (2018) 17:2349-59. doi: 10.1080/ 15384101.2018.1526599

56. Cao S, Wang G, Wang J, Li C, Zhang L. Hsa_circ_101280 promotes hepatocellular carcinoma by regulating miR-375/JAK2. Immunol Cell Biol (2019) 97:218-28. doi: 10.1111/imcb.12213

57. Guan Z, Tan J, Gao W, Li X, Yang Y, Li X, et al. Circular RNA hsa_circ_0016788 regulates hepatocellular carcinoma tumorigenesis through miR-486/CDK4 pathway. J Cell Physiol (2018) 234:500-8. doi: $10.1002 /$ jcp. 26612

58. Cai H, Hu B, Ji L, Ruan X, Zheng Z. Hsa_circ_0103809 promotes cell proliferation and inhibits apoptosis in hepatocellular carcinoma by targeting miR-490-5p/SOX2 signaling pathway. Am J Transl Res (2018) 10:1690-702.

59. Jia C, Yao Z, Lin Z, Zhao L, Cai X, Chen S, et al. circNFATC3 sponges miR548I acts as a ceRNA to protect NFATC3 itself and suppressed hepatocellular carcinoma progression. J Cell Physiol (2020) 236:1252-69. doi: 10.1002/ jcp.29931

60. Wang J, Tan Q, Wang W, Yu J. Mechanism of the Regulatory Effect of Overexpression of circMTO1 on Proliferation and Apoptosis of Hepatoma Cells via miR-9-5p/NOX4 Axis. Cancer Manag Res (2020) 12:3915-25. doi: 10.2147/CMAR.S240719

61. Chen W, Quan Y, Fan S, Wang H, Liang J, Huang L, et al. Exosometransmitted circular RNA hsa_circ_0051443 suppresses hepatocellular carcinoma progression. Cancer Lett (2020) 475:119-28. doi: 10.1016/ j.canlet.2020.01.022

62. Shi L, Yan P, Liang Y, Sun Y, Shen J, Zhou S, et al. Circular RNA expression is suppressed by androgen receptor (AR)-regulated adenosine deaminase that acts on RNA (ADAR1) in human hepatocellular carcinoma. Cell Death Dis (2017) 8:e3171. doi: 10.1038/cddis.2017.556

63. Yu J, Xu QG, Wang ZG, Yang Y, Zhang L, Ma JZ, et al. Circular RNA cSMARCA5 inhibits growth and metastasis in hepatocellular carcinoma. J Hepatol (2018) 68:1214-27. doi: 10.1016/j.jhep.2018.01.012

64. Qiu L, Huang Y, Li Z, Dong X, Chen G, Xu H, et al. Circular RNA profiling identifies circADAMTS13 as a miR-484 sponge which suppresses cell proliferation in hepatocellular carcinoma. Mol Oncol (2019) 13:441-55. doi: 10.1002/1878-0261.12424

65. Song C, Li D, Liu H, Sun H, Liu Z, Zhang L, et al. The competing endogenous circular RNA ADAMTS14 suppressed hepatocellular carcinoma progression through regulating microRNA-572/regulator of calcineurin 1. J Cell Physiol (2019) 234:2460-70. doi: 10.1002/jcp.26764

66. Zhong L, Wang Y, Cheng Y, Wang W, Lu B, Zhu L, et al. Circular RNA circC3P1 suppresses hepatocellular carcinoma growth and metastasis through miR-4641/PCK1 pathway. Biochem Biophys Res Commun (2018) 499:1044-9. doi: 10.1016/j.bbrc.2018.03.221

67. Wang Z, Zhao Y, Wang Y, Jin C. Circular RNA circHIAT1 inhibits cell growth in hepatocellular carcinoma by regulating miR-3171/PTEN axis. BioMed Pharmacother (2019) 116:108932. doi: 10.1016/j.biopha.2019.108932

68. Chen Z, Zuo X, Pu L, Zhang Y, Han G, Zhang L, et al. circLARP4 induces cellular senescence through regulating miR-761/RUNX3/p53/p21 signaling in hepatocellular carcinoma. Cancer Sci (2019) 110:568-81. doi: 10.1111/ cas. 13901

69. Xu L, Feng X, Hao X, Wang $\mathrm{P}$, Zhang $\mathrm{Y}$, Zheng X, et al. CircSETD3 (Hsa_circ_0000567) acts as a sponge for microRNA-421 inhibiting hepatocellular carcinoma growth. J Exp Clin Cancer Res (2019) 38:98. doi: 10.1186/s13046-019-1041-2

70. Zhang PF, Wei CY, Huang XY, Peng R, Yang X, Lu JC, et al. Circular RNA circTRIM33-12 acts as the sponge of MicroRNA-191 to suppress hepatocellular carcinoma progression. Mol Cancer (2019) 18:105. doi: 10.1186/s12943-019-1031-1

71. Li X, Shen M. Circular RNA hsa_circ_103809 suppresses hepatocellular carcinoma proliferation and invasion by sponging miR-620. Eur Rev Med Pharmacol Sci (2019) 23:555-66. doi: 10.26355/eurrev_201902_16868

72. Xiong DD, Feng ZB, Lai ZF, Qin Y, Liu LM, Fu HX, et al. High throughput circRNA sequencing analysis reveals novel insights into the mechanism of nitidine chloride against hepatocellular carcinoma. Cell Death Dis (2019) 10:658. doi: 10.1038/s41419-019-1890-9

73. Matboli M, Shafei AE, Ali MA, Ashry AM, Kamal KM, Agag MA, et al. circRNAs (hsa_circ_00156, hsa_circ_000224, and hsa_circ_000520) are novel potential biomarkers in hepatocellular carcinoma. J Cell Biochem (2018). doi: 10.1002/jcb.28045

74. Zhang C, Zhang C, Lin J, Wang H. Circular RNA Hsa_Circ_0091579 Serves as a Diagnostic and Prognostic Marker for Hepatocellular Carcinoma. Cell Physiol Biochem (2018) 51:290-300. doi: 10.1159/000495230

75. Chen D, Zhang C, Lin J, Song X, Wang H. Screening differential circular RNA expression profiles reveal that hsa_circ_0128298 is a biomarker in the diagnosis and prognosis of hepatocellular carcinoma. Cancer Manag Res (2018) 10:1275-83. doi: 10.2147/CMAR.S166740

76. Wang X, Zhang C, Wu Z, Chen Y, Shi W. CircIBTK inhibits DNA demethylation and activation of AKT signaling pathway via miR-29b in peripheral blood mononuclear cells in systemic lupus erythematosus. Arthritis Res Ther (2018) 20:118. doi: 10.1186/s13075-018-1618-8

77. Zhou C, Molinie B, Daneshvar K, Pondick JV, Wang J, Van Wittenberghe N, et al. Genome-Wide Maps of m6A circRNAs Identify Widespread and CellType-Specific Methylation Patterns that Are Distinct from mRNAs. Cell Rep (2017) 20:2262-76. doi: 10.1016/j.celrep.2017.08.027

78. Wu C, Deng L, Zhuo H, Chen X, Tan Z, Han S, et al. Circulating circRNA predicting the occurrence of hepatocellular carcinoma in patients with $\mathrm{HBV}$ infection. J Cell Mol Med (2020) 24:10216-22. doi: 10.1111/jcmm.15635

79. Cheng F, Wang L, Zhang J. Circular RNA 0016788 displays as a biomarker for tumor progression and poor prognosis in surgical hepatocellular carcinoma patients. J Clin Lab Anal (2020) 34:e23300. doi: 10.1002/jcla.23300

80. Zhang X, Xu Y, Qian Z, Zheng W, Wu Q, Chen Y, et al. circRNA_104075 stimulates YAP-dependent tumorigenesis through the regulation of HNF4a and may serve as a diagnostic marker in hepatocellular carcinoma. Cell Death Dis (2018) 9:1091. doi: 10.1038/s41419-018-1132-6

81. Sun XH, Wang YT, Li GF, Zhang N, Fan L. Serum-derived three-circRNA signature as a diagnostic biomarker for hepatocellular carcinoma. Cancer Cell Int (2020) 20:226. doi: 10.1186/s12935-020-01302-y

82. Lei B, Zhou J, Xuan X, Tian Z, Zhang M, Gao W, et al. Circular RNA expression profiles of peripheral blood mononuclear cells in hepatocellular carcinoma patients by sequence analysis. Cancer Med (2019) 8:1423-33. doi: $10.1002 / \mathrm{cam} 4.2010$ 
83. Pan H, Tang L, Jiang H, Li X, Wang R, Gao J, et al. Enhanced expression of circ_0000267 in hepatocellular carcinoma indicates poor prognosis and facilitates cell progression by sponging miR-646. J Cell Biochem (2019). doi: $10.1002 /$ jcb. 28411

84. Wang H, Chen W, Jin M, Hou L, Chen X, Zhang R, et al. CircSLC3A2 functions as an oncogenic factor in hepatocellular carcinoma by sponging miR-490-3p and regulating PPM1F expression. Mol Cancer (2018) 17:165. doi: 10.1186/s12943-018-0909-7

85. Weng Q, Chen M, Li M, Zheng YF, Shao G, Fan W, et al. Global microarray profiling identified hsa_circ_0064428 as a potential immune-associated prognosis biomarker for hepatocellular carcinoma. J Med Genet (2019) 56:32-8. doi: 10.1136/jmedgenet-2018-105440

86. Zhou S, Wei J, Wang Y, Liu X. Cisplatin resistance-associated circRNA_ 101237 serves as a prognostic biomarker in hepatocellular carcinoma. Exp Ther Med (2020) 19:2733-40. doi: 10.3892/etm.2020.8526

87. Huang W, Huang F, Feng C. CircFoxo3 Promotes Adriamycin Resistance Through Regulation of miR-199a-5p/ATP Binding Cassette Subfamily C Member 1 Axis in Hepatocellular Carcinoma. Onco Targets Ther (2020) 13:5113-22. doi: 10.2147/OTT.S243571
88. Zhang PF, Gao C, Huang XY, Lu JC, Guo XJ, Shi GM, et al. Cancer cellderived exosomal circUHRF1 induces natural killer cell exhaustion and may cause resistance to anti-PD1 therapy in hepatocellular carcinoma. Mol Cancer (2020) 19:110. doi: 10.1186/s12943-020-01222-5

89. Chen H, Liu S, Li M, Huang P, Li X. circ_0003418 Inhibits Tumorigenesis And Cisplatin Chemoresistance Through Wnt/beta-Catenin Pathway In Hepatocellular Carcinoma. Onco Targets Ther (2019) 12:9539-49. doi: 10.2147/OTT.S229507

Conflict of Interest: The authors declare that the research was conducted in the absence of any commercial or financial relationships that could be construed as a potential conflict of interest.

Copyright (C) 2021 Xiong, He, Dang, Wu, Feng and Chen. This is an open-access article distributed under the terms of the Creative Commons Attribution License (CC BY). The use, distribution or reproduction in other forums is permitted, provided the original author(s) and the copyright owner(s) are credited and that the original publication in this journal is cited, in accordance with accepted academic practice. No use, distribution or reproduction is permitted which does not comply with these terms. 